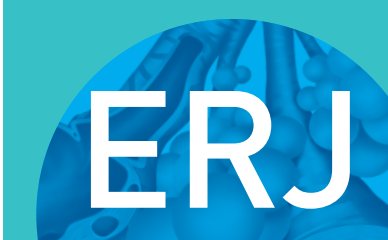

open research

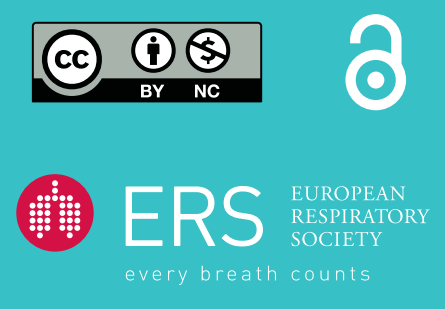

\section{Detection of Cutibacterium acnes in granulomas of patients with either hypersensitivity pneumonitis or vasculitis reveals that its presence is not unique for sarcoidosis}

\author{
To the Editor:
}

Granulomas are compact organised structures of different immune cells, including macrophages, lymphocytes and plasma cells, thought to be formed when (foreign) antigens cannot be cleared. The differential diagnosis of a granulomatous lesion is broad and includes infectious aetiologies, malignancy and inflammatory disorders like vasculitis, hypersensitivity pneumonitis and sarcoidosis [1].

Cutibacterium acnes, formerly Propionibacterium acnes, is a Gram-positive bacterium that is a commensal of the skin [2]. Studies have demonstrated that C. acnes is also able to induce granulomas [3]. Furthermore, C. acnes is suggested to be involved in the pathogenesis of sarcoidosis, a complex inflammatory disease mainly involving the lungs and lymph nodes, characterised by noncaseating granulomas [4].

Although presence of $C$. acnes has already been shown in granulomas of sarcoidosis patients [5], it is unknown whether this bacterium can also be found in granulomatous disorders with frequent involvement of the lungs other than sarcoidosis, such as hypersensitivity pneumonitis (HP), granulomatosis with polyangiitis (GPA) and eosinophilic granulomatosis with polyangiitis (EGPA).

To investigate whether the presence of $C$. acnes in granulomas is specific to sarcoidosis, we examined granulomatous tissue of HP, GPA and EGPA patients for the presence of C. acnes.

Tissue blocks were collected from patients with HP, GPA or EGPA who participated in our biobank study. Patients were included in the study when enough residual tissue was available and when granulomas could be detected in the haematoxylin-stained tissue sections. Tissue blocks of 35 patients with HP and of 13 patients with (E)GPA were collected. Tissue blocks of all HP and nine (E)GPA patients showed granulomas and had enough tissue left to be included in the study. The study was approved by the Medical Research Ethics Committees United of the St Antonius Hospital (R05-08A) and written consent was obtained from all patients. Formalin-fixed, paraffin-embedded tissue sections were immunohistochemically stained with the PAB antibody, a C. acnes-specific monoclonal antibody that binds to the cell membrane-bound lipoteichoic acid (LTA) of the bacterium [5]. Full methods are described elsewhere [6]. Differences in the presence of C. acnes between the patient groups was compared using the Chi-squared test. An independent-sample t-test was used to compare bronchoalveolar lavage (BAL) lymphocytes between HP patients. Fisher's exact test was used to compare presence of C. acnes in granulomas of HP patients with and without a known inducing agent. p-values $<0.05$ were considered significant.

@ERSpublications

Presence of $C$. acnes in granulomas is not unique to sarcoidosis but can also be found in patients with HP or EGPA. C. acnes may be involved in the pathogenesis of those granulomatous diseases in a mitogenic way. https://bit.ly/3pUOPeC

Cite this article as: Beijer E, Seldenrijk K, Meek B, et al. Detection of Cutibacterium acnes in granulomas of patients with either hypersensitivity pneumonitis or vasculitis reveals that its presence is not unique for sarcoidosis. ERJ Open Res 2021; 7: 00930-2020 [https://doi.org/10.1183/ 23120541.00930-2020].

Copyright $\odot$ The authors 2021. This version is distributed under the terms of the Creative Commons Attribution NonCommercial Licence 4.0. For commercial reproduction rights and permissions contact permissions@ersnet.org 
a)

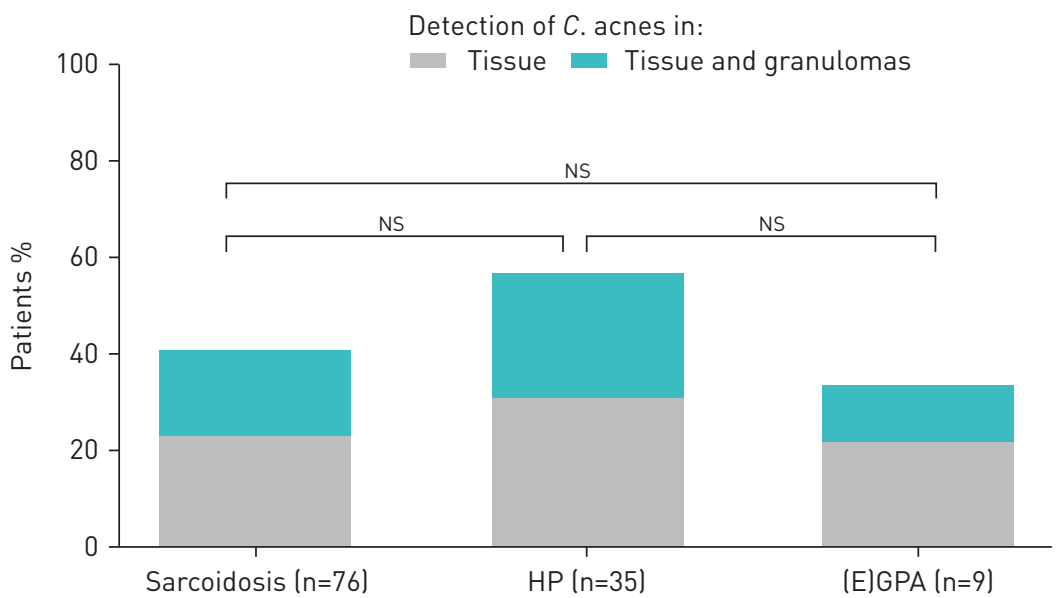

\begin{tabular}{lccc}
\hline Age years mean \pm SD & $44.0 \pm 12.2$ & $58.2 \pm 9.2$ & $51.6 \pm 14.7$ \\
Male sex & $42(55.3)$ & $17(48.6)$ & $6(66.7)$ \\
Origin of tissue & & & \\
$\quad$ Lung & $30(39.5)$ & $34(97.1)$ & $6(66.7)$ \\
Lymph node & $24(31.6)$ & $1(2.9)$ & $1(11.1)$ \\
Skin & $17(22.4)$ & & $2(22.2)$ \\
Nose & $1(1.3)$ & & \\
Other & $4(5.3)$ & & \\
Inducing agent & & $14(40.0)$ & $\mathrm{NA}$ \\
Birds & $\mathrm{NA}$ & $3(8.6)$ & \\
Farmer's lung & & $18(51.4)$ & \\
Unknown & & & \\
\hline
\end{tabular}
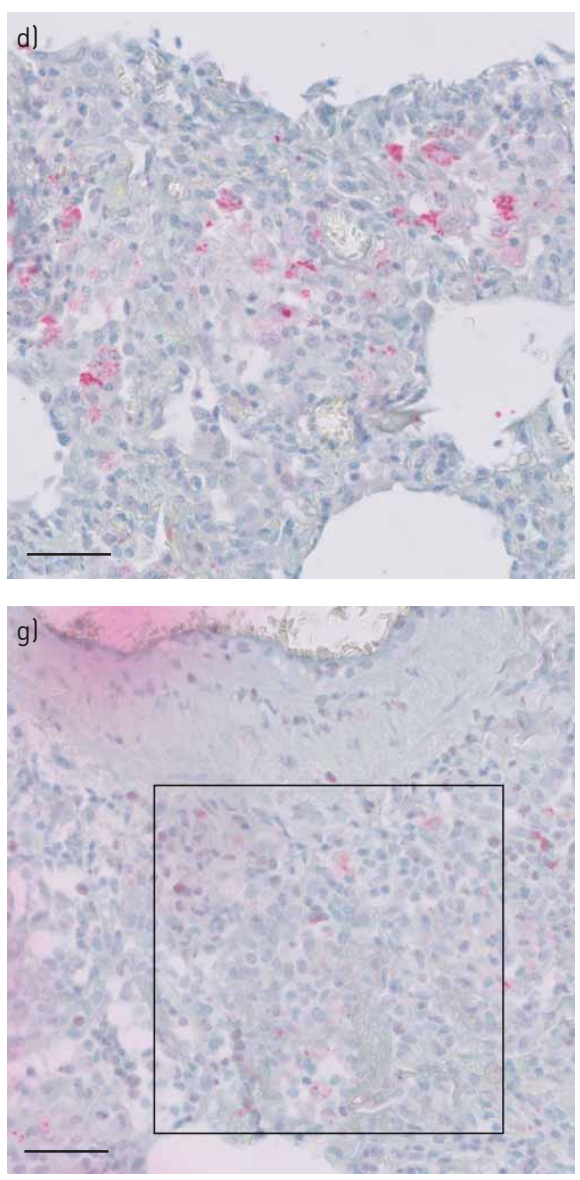
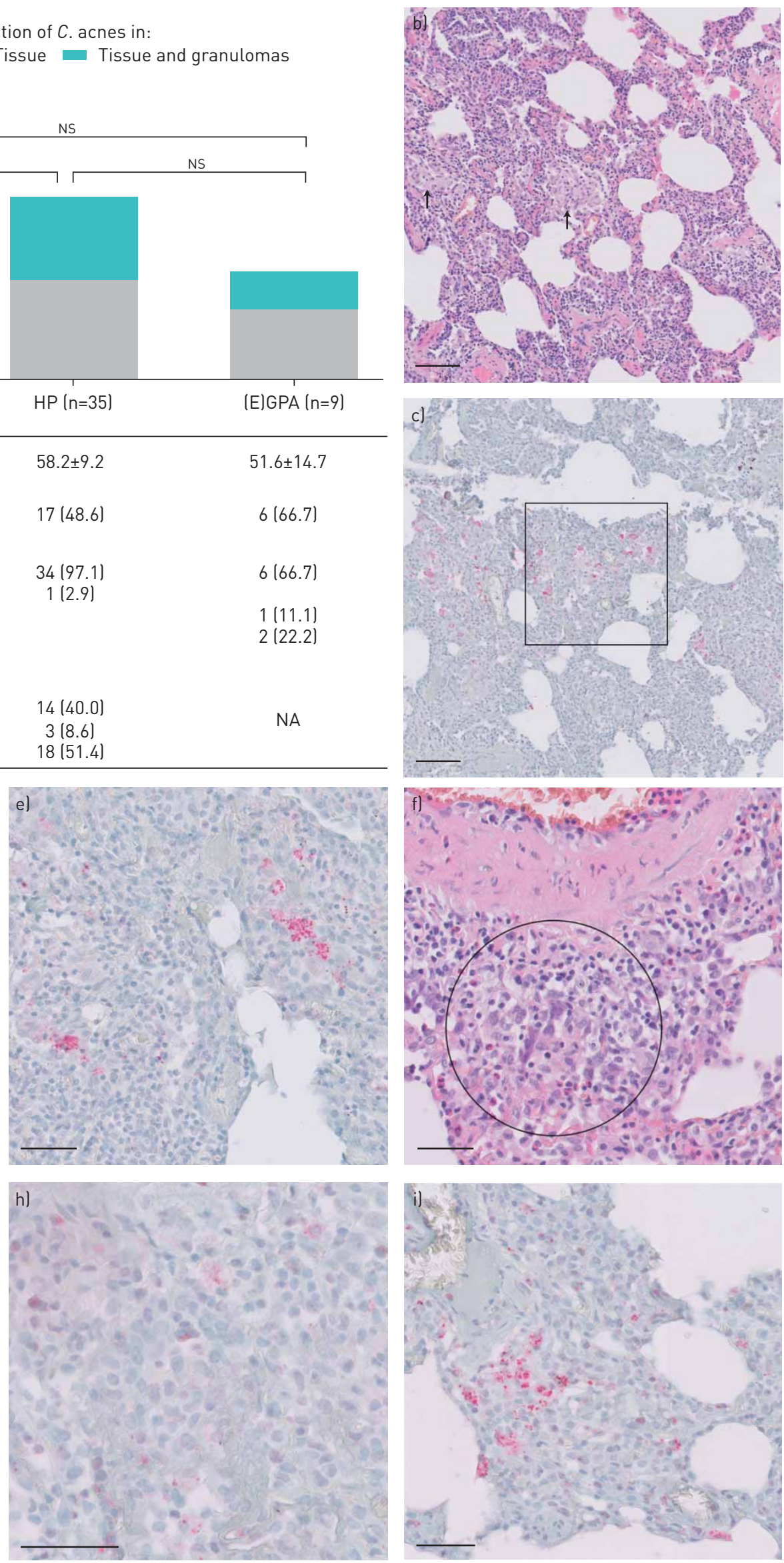

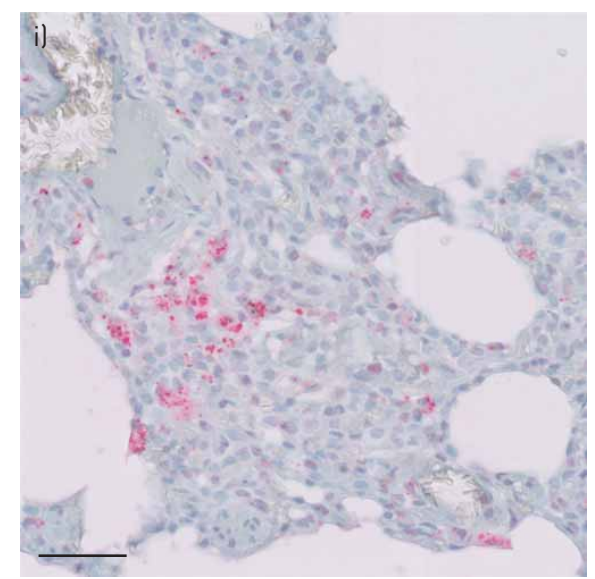


FIGURE 1 Presence of Cutibacterium acnes in tissue and granulomas of patients with hypersensitivity pneumonitis (HP) and (eosinophilic) granulomatosis with polyangiitis ((E)GPA). a) Characteristics of all included patients and percentage of patients with presence of $C$. acnes in tissue or in tissue and granulomas. The sarcoidosis group was previously described [6]. No significant difference was observed between the three groups regarding $C$. acnes in tissue or $C$. acnes in granulomas ( $p=0.210$ and $p=0.460$, respectively). Data are presented as $n$ (\%) unless otherwise stated. b) Haematoxylin and eosin stain (HE) stain of lung tissue from an HP patient. The black arrows show granulomas. Scale bar=100 $\mu$ m. c) Positive PAB immunohistochemistry (red) in the granulomas corresponding to (b). Scale bar=100 $\mu \mathrm{m}$. d) Higher-magnification image corresponding to the square in picture (c). Scale bar=50 $\mu \mathrm{m}$. e) Area of positive PAB immunohistochemistry outside granulomas. Scale bar=50 $\mu \mathrm{m}$. f) HE stain of lung tissue from an EGPA patient, with an immature granuloma indicated by the circle. Scale bar=50 $\mu$ m. $g$ ) Positive PAB immunohistochemistry in the granuloma corresponding to (f). Scale bar $=50 \mu \mathrm{m}$. h) Higher-magnification image corresponding to the square in (g). Scale bar=50 $\mu \mathrm{m}$. i) Area of positive PAB immunohistochemistry outside granulomas. Scale bar=50 $\mu \mathrm{m}$. Ns: nonsignificant; NA: not applicable.

Presence of C. acnes in tissue was found in $57.1 \%$ of HP patients (20 out of 35) and in 33.3\% of (E)GPA patients (three out of nine). More specifically, the presence of $C$. acnes was observed inside granulomas of 25.7\% HP patients (nine out of 35) and of 11.1\% (E)GPA patients (one out of nine). Results between the diagnostic groups were not statistically significant $(\mathrm{p}=0.272$ and $\mathrm{p}=0.659$ for presence of $C$. acnes in tissue and granulomas, respectively). Moreover, the percentages of patients with presence of C. acnes in tissue or granulomas was comparable with a previously described Dutch sarcoidosis cohort (figure 1). As the presence of $C$. acnes appeared not to be specific to sarcoidosis and considering the known attributed mitogenic properties of this bacterium [7], we further explored such a role for C. acnes in our study by assessing BAL lymphocytosis in the patients with HP. Interestingly, a higher percentage of lymphocytes was indeed observed in HP patients that stained positively for C. acnes in granulomas compared to HP patients without $C$. acnes staining in granulomas $(70.3 \%$ versus $41.3 \%, \mathrm{p}=0.018$ ). The percentage of patients with presence of $C$. acnes in granulomas did not significantly differ between the HP group in which an inducing agent was known compared to the HP group in which the inducing agent was unknown (35.3\% versus $16.7 \%, \mathrm{p}=0.264)$.

Up to now, considering granulomatous diseases, C. acnes has solely been related to the pathogenesis of sarcoidosis [8]. Presence of C. acnes has been demonstrated in tissue and granulomas of Japanese, German and Dutch sarcoidosis patients $[5,6]$. To the best of our knowledge, this is the first study that demonstrates that $C$. acnes can also be detected in tissue and granulomas of patients with HP and vasculitis. If the presence of $C$. acnes is not disease specific, as our data suggest, one can debate whether C. acnes has an antigenic role in sarcoidosis pathogenesis. Regarding HP and (E)GPA pathogenesis, no data are available suggesting an antigenic role for $C$. acnes. However, data on a possible mitogenic role for C. acnes have been described [7,9]. The higher percentage of lymphocytes observed in BAL of HP patients with presence of $C$. acnes in granulomas, supports the hypothesis that $C$. acnes may indeed act as a mitogen by enhancing lymphocyte proliferation. Moreover, since we observed presence of $C$. acnes in granulomas of HP patients in which a causal agent was previously identified, it is unlikely that C. acnes act as a specific agent in HP.

The PAB antibody that was used in this study reacts with the cell membrane-bound LTA of the C. acnes bacteria. LTA is a cell wall polymer of Gram-positive bacteria and plays a role in bacterial growth, membrane homeostasis and virulence [10]. Furthermore, LTAs have shown to be immunogenic [11] and activate the innate immune system via toll-like receptor 2 (TLR2) and NOD-like receptor family pyrin domain-containing 6 (NLRP6) [12, 13], key receptor families involved in the innate immune defence against invading pathogens. Interleukin-18 production can be stimulated following activation of NLRP6 [13]. Results of several studies suggest that TLR2 and interleukin-18 are at least partly involved in granuloma formation $[14,15]$, and in vitro and in vivo models have indeed shown that C. acnes is able to induce granulomas [3]. It is therefore possible that C. acnes is not a specific trigger of sarcoidosis, HP and (E)GPA, but that its LTA contributes to inflammation and granuloma formation.

A limitation of the study is that no control group with healthy individuals was included in the study. It is, however, difficult to include an appropriate control group in the study, as healthy controls would usually not show granulomas in tissue. Another limitation was that we have not been able to analyse the disease course in HP patients due to the small sample size in combination with too many missing follow-up data. Furthermore, the sample size of vasculitis patients from whom suitable tissue was available was too small to perform a subanalysis on disease course or BAL. Consequently, we were unable to examine whether C. acnes is related to a chronic disease course in (E)GPA and HP as well. Due to the low sample sizes of the HP and EGPA groups, the power to detect a difference with the sarcoidosis group was too small to conclude that those diseases, and HP patients with and without a known inducing agent, do not really differ regarding presence of $C$. acnes in granulomas. Although the percentages of $C$. acnes in granulomas of the nonsarcoidosis group were quite comparable to the sarcoidosis group, further studies using a higher number of patients are needed to clarify whether there is really no difference in presence of $C$. acnes between those diseases. Last, in this study we only examined LTA of the C. acnes bacteria. It is plausible 
that LTAs of other Gram-positive bacteria can also be found in granulomas. Future studies will have to determine whether granulomas are uniquely related to LTAs of $C$. acnes or can be attributed to LTAs in general.

To conclude, we have shown that presence of $C$. acnes in granulomas is not unique for sarcoidosis but can also be found in patients with HP and EGPA. We hypothesise that C. acnes may be involved in the disease pathogenesis of those granulomatous diseases in a mitogenic way. Future studies are needed to determine the precise role of $C$. acnes and other LTAs in those granulomatous diseases.

\section{Els Beijer $\oplus^{1}$, Kees Seldenrijk ${ }^{2}$, Bob Meek $^{3}$, Jan Damen ${ }^{4}$, Marian J.R. Quanjel ${ }^{1}$, Jan C. Grutters ${ }^{1,5}$ and Marcel Veltkamp ${ }^{1,5}$ \\ ${ }^{1}$ Interstitial Lung Diseases Centre of Excellence, Dept of Pulmonology, St Antonius Hospital, Nieuwegein, The Netherlands. ${ }^{2}$ Pathology DNA, Dept of Pathology, St Antonius Hospital, Nieuwegein, The Netherlands. ${ }^{3}$ Dept of Medical Microbiology and Immunology, St Antonius Hospital, Nieuwegein, The Netherlands. ${ }^{4}$ Pathology DNA, Dept of Pathology, Jeroen Bosch Hospital, 's-Hertogenbosch, The Netherlands. ${ }^{5}$ Division of Heart and Lungs, University Medical Centre, Utrecht, The Netherlands.}

Correspondence: Marcel Veltkamp, ILD research, Koekoekslaan 1, 3435 CM Nieuwegein, The Netherlands. E-mail: m.veltkamp@antoniusziekenhuis.nl

Received: 11 Dec 2020 | Accepted: 29 Jan 2021

Acknowledgements: We would like to thank the research group of Prof. Y. Eishi (Dept of Human Pathology, Tokyo Medical and Dental University Graduate school, Tokyo, Japan) who kindly provided the PAB antibody.

Author contributions: E. Beijer contributed to data acquisition and analysis, statistical analysis, and drafting and editing of the manuscript. K. Seldenrijk participated in the design of the study, scored the staining results and edited the manuscript. B. Meek edited the manuscript. J. Damen carried out all the immunohistochemical staining and developed the automated staining protocol. M.J.R. Quanjel contributed to data acquisition and edited the manuscript. J.C. Grutters supervised the project and edited the manuscript. M. Veltkamp designed the study, supervised the project and edited the manuscript.

Conflict of interest: None declared.

Support statement: This study is part of the TopZorg grant funded by ZonMw (number 10070012010004). This funder was not involved in the study design; in the collection, analysis and interpretation of the data; in the writing of the report; or in the decision to submit the paper for publication. Funding information for this article has been deposited with the Crossref Funder Registry.

\section{References}

1 Shah KK, Pritt BS, Alexander MP. Histopathologic review of granulomatous inflammation. J Clin Tuberc Other Mycobact Dis 2017; 7: 1-12.

2 Leheste JR, Ruvolo KE, Chrostowski JE, et al. P. acnes-driven disease pathology: current knowledge and future directions. Front Cell Infect Microbiol 2017; 7: 81.

3 Iio K, Iio $\mathrm{T}$, Okui $\mathrm{Y}$, et al. Experimental pulmonary granuloma mimicking sarcoidosis induced by Propionibacterium acnes in mice. Acta Med Okayama 2010; 64: 75-83.

4 Grunewald J, Grutters JC, Arkema EV, et al. Sarcoidosis. Nat Rev Dis Prim 2019; 5: 45.

5 Negi M, Takemura T, Guzman J, et al. Localisation of Propionibacterium acnes in granulomas supports a possible etiologic link between sarcoidosis and the bacterium. Mod Pathol 2012; 25: 1284-1297.

6 Beijer E, Seldenrijk K, Eishi Y, et al. Presence of Propionibacterium acnes in granulomas associates with a chronic disease course in Dutch sarcoidosis patients. ERJ Open Res 2020; 7: 486-2020.

7 Jappe U, Ingham E, Henwood J, et al. Propionibacterium acnes and inflammation in acne; $P$. acnes has T-cell mitogenic activity. Br J Dermatol 2002; 146: 202-209.

8 Eishi Y. Etiologic link between sarcoidosis and Propionibacterium acnes. Respir Investig 2013; 51: 56-68.

9 Yeon SJ, Matsumoto SE, Yamashita M, et al. Propionibacterium acnes acts as an adjuvant in in vitro immunisation of human peripheral blood mononuclear cells. Biosci Biotechnol Biochem 2007; 71: 1963-1969.

10 Percy MG, Gründling A. Lipoteichoic acid synthesis and function in gram-positive bacteria. Annu Rev Microbiol 2014; 68: 81-100.

11 Knox KW, Wicken AJ. Immunological properties of teichoic acids. Bacteriol Rev 1973; 37: $215-257$.

12 Mitchell JA, Paul-Clark MJ, Clarke GW, et al. Critical role of toll-like receptors and nucleotide oligomerisation domain in the regulation of health and disease. J Endocrinol 2007; 193: 323-330.

13 Hara H, Seregin SS, Yang D, et al. The NLRP6 inflammasome recognises lipoteichoic acid and regulates gram-positive pathogen infection. Cell 2018; 175: 1651-1664.

14 Hu W, Yang S, Shimada Y, et al. Infection and RNA-seq analysis of a zebrafish tlr2 mutant shows a broad function of this toll-like receptor in transcriptional and metabolic control and defense to Mycobacterium marinum infection. BMC Genom 2019; 20.

15 Volkman DJ. Sarcoidosis and IL18. Clin Immunol 2001; 101: 1-2. 